\title{
A multiple model adaptive architecture for the state estimation in discrete-time uncertain LPV systems
}

\author{
Damiano Rotondo, Vahid Hassani, Andrea Cristofaro
}

\begin{abstract}
This paper addresses the problem of multiple model adaptive estimation (MMAE) for discrete-time linear parameter varying (LPV) systems that are affected by parametric uncertainty. The MMAE system relies on a finite number of local observers, each designed using a selected model (SM) from the set of possible plant models. Each local observer is an LPV Kalman filter, obtained as a linear combination of linear time invariant (LTI) Kalman filters. It is shown that if some suitable distinguishability conditions are fulfilled, the MMAE will identify the SM corresponding to the local observer with smallest output prediction error energy. The convergence of the unknown parameter estimation, and its relation with the varying parameters, are discussed. Simulation results illustrate the application of the proposed method.
\end{abstract}

\section{INTRODUCTION}

The design of state observers is an important control problem, due to its importance in feedback control and process monitoring systems. However, the uncertainty coming from the mismatch between the model and the real plant impedes an accurate estimation of the state variables, which is often a fundamental requirement in many applications [1]. In these cases, the design of a conventional state estimator that converges to the real state value cannot be realized [2].

For this reason, a problem that has been investigated with attention in recent years is to provide alternatives for estimating the state in uncertain systems. In this sense, a proposed solution is to construct an observer that estimates the set of admissible values for the state at each instant of time, i.e. an interval observer [3]-[5]. Another proposed solution is to use adaptive estimation algorithms [6]-[8], which perform an adaptation w.r.t. the uncertainty in the plant parameters. Among these algorithms, the multiple model adaptive estimation (MMAE) has demonstrated to be a powerful approach, which has been employed successfully in several occasions [9]-[13]. In this approach, a set of models is selected/designed to represent the possible system behavior patterns, and the overall estimate is obtained by combining

This work has been supported by the Research Council of Norway through the Centres of Excellence funding scheme (ref. 223254 - NTNU AMOS). D. Rotondo is also supported by the ERCIM Alain Bensoussan Fellowship programme.

D. Rotondo is with Automatic Control Department, Universitat Politècnica de Catalunya (UPC), Rambla de Sant Nebridi, 11, 08222 Terrassa, Spain, and with Department of Engineering Cybernetics (ITK), Norwegian University of Science and Technology (NTNU), O. S. Bragstad Plass 2D, 7014 Trondheim, Norway. V. Hassani is with Department of Marine Technology, NTNU, NO 7491 Trondheim, Norway, and with SINTEF Ocean (formerly known as MARINTEK). A. Cristofaro is with ITK - NTNU, and with School of Science and Technology, University of Camerino, via Madonna delle Carceri, 62302 Camerino, Italy. e-mail: \{damiano.rotondo\}@yahoo.it; \{vahid.hassani\}@ntnu.no; andrea.cristofaro\}@unicam.it the estimates from the filters running in parallel, based on the individual models that match these patterns [14].

With the aim of developing simple and yet efficient solutions to the analysis and control of nonlinear systems, linear parameter varying (LPV) methodologies have been applied successfully by extending linear time invariant (LTI) techniques [15]. LPV models are linear state space models whose matrices depend on time-varying parameters [16]. The LPV framework is suitable to deal with nonlinear systems, since they can be brought to the so-called quasi-LPV form, in which the parameters are functions of endogenous signals such as states, inputs and outputs [17]-[20]. Nowadays, LPV methodologies are theoretically well founded and find applications in several fields, such as aviation [21], robotics [22] and automotive [23].

In this work, we develop an LPV MMAE for estimating the state and the value of the unknown parameters in discrete time uncertain LPV plants. The proposed LPV MMAE comprises a collection of local observers, each of which provides the state estimation which would correspond to a predefined value of the unknown parameters. We show that under some suitable condition, the identified unknown parameters will correspond to the observer that exhibits the smallest output prediction error energy. The convergence of the unknown parameter estimation, and its relation with the varying parameters, are discussed thoroughly, and the concept of undecidable sets is introduced as well.

The structure of the paper is as follows. Section II introduces the LPV MMAE, and Section III summarizes its relevant properties. Section IV provides a discussion of the convergence of the parameter estimation. Section V illustrates the proposed technique using simulations. Finally, Section VI summarizes the main conclusions.

\section{LPV Multiple Model Adaptive Estimator}

We consider discrete time multiple input multiple output (MIMO) linear parameter varying (LPV) models of the form:

$$
\begin{aligned}
x(t+1) & =A(\kappa(t), \theta(t)) x(t)+B(\kappa(t), \theta(t)) u(t) \\
& +G(\kappa(t), \theta(t)) w(t) \\
y(t)= & C(\kappa(t), \theta(t)) x(t)+v(t)
\end{aligned}
$$

where $x \in \mathbb{R}^{n}$ denotes the state vector, $u \in \mathbb{R}^{m}$ is the control input, $w \in \mathbb{R}^{r}$ is a disturbance that cannot be measured, and $y \in \mathbb{R}^{q}$ is the measured output, which is affected by the measurement noise $v \in \mathbb{R}^{q}$. The initial condition $x(0)$ is assumed to be unknown, while the signals $w(t), v(t)$ are uncorrelated and described as white noises with power 
spectral density (PSD) $S_{w}$ and $S_{v}$, respectively. The matrix functions $A(\cdot), B(\cdot), C(\cdot)$ and $G(\cdot)$ depend on both an unknown parameter $\kappa(t)$ and a known varying parameter vector $\theta(t) \in \Theta \subset \mathbb{R}^{s}$. In order to ease the notation, $A_{\mathcal{K}}(\theta(t))$, $B_{\kappa}(\theta(t)), C_{\kappa}(\theta(t)), G_{\kappa}(\theta(t))$ will be used alternatively to denote the matrix functions appearing in (1)-(2).

In order to estimate the state, a finite set of candidate parameter values $\left\{\kappa_{1}, \ldots, \kappa_{N}\right\}$ indexed by $i \in\{1, \ldots, N\}$ is considered. It is worth remarking that, contrarily to the case of switched systems [24], it is not requested that the parameter $\kappa(t)$ varies within the set $\left\{\kappa_{1}, \ldots, \kappa_{N}\right\}$. It is assumed that for each candidate parameter value $\kappa_{i}, i \in$ $\{1, \ldots, N\}$, the corresponding matrix functions $A_{\kappa_{i}}(\theta(t))$, $B_{\kappa_{i}}(\theta(t)), C_{\kappa_{i}}(\theta(t))$ and $G_{\kappa_{i}}(\theta(t))$ are polytopic [25], i.e. they can be described as the convex sum of $L$ constant matrices denoted as vertex matrices:

$$
\left(\begin{array}{cc}
A_{\kappa_{i}}(\theta(t)) & B_{\kappa_{i}}(\theta(t)) \\
C_{\kappa_{i}}(\theta(t)) & G_{\kappa_{i}}(\theta(t))
\end{array}\right)=\sum_{j=1}^{L} h_{i}^{(j)}(\theta(t))\left(\begin{array}{cc}
A_{\kappa_{i}}^{(j)} & B_{\kappa_{i}}^{(j)} \\
C_{\kappa_{i}}^{(j)} & G_{\kappa_{i}}^{(j)}
\end{array}\right)
$$

where, for a given $i$, the coefficients $h_{i}^{(j)}(\theta(t))$ satisfy the following property:

$$
\sum_{j=1}^{L} h_{i}^{(j)}(\theta)=1, \quad h_{i}^{(j)}(\theta) \in[0,1] \quad \forall \theta \in \Theta
$$

Hence, inspired by the LTI multiple model adaptive estimator (MMAE) described in [26], the following LPV MMAE is proposed:

$$
\begin{aligned}
\hat{x}(t) & =\sum_{i=1}^{N} p_{i}(t) \hat{x}\left(t \mid \kappa_{i}\right) \\
\hat{y}(t) & =\sum_{i=1}^{N} p_{i}(t) \hat{y}\left(t \mid \kappa_{i}\right) \\
\hat{\kappa}(t) & =\kappa_{i^{*}(t)}, \quad i^{*}(t)=\arg \max _{i \in\{1, \ldots, N\}} p_{i}(t)
\end{aligned}
$$

where $\hat{x}(t), \hat{y}(t)$ and $\hat{\kappa}(t)$ are the estimates of the state $x(t)$, the output $y(t)$, and the unknown parameter vector $\kappa$, respectively, and $p_{i}(t)$ are dynamic weights, that will be defined in the following. In (5)-(6), each $\hat{x}\left(t \mid \kappa_{i}\right), \hat{y}\left(t \mid \kappa_{i}\right)$ correspond to a local state estimation, obtained through the following LPV Kalman filter [27]:

$$
\begin{aligned}
& \hat{x}\left(t \mid \kappa_{i}\right)=\sum_{j=1}^{L} \hat{x}_{j}\left(t \mid \kappa_{i}\right) \\
& \hat{y}\left(t \mid \kappa_{i}\right)=C_{\kappa_{i}}(\theta(t)) \hat{x}\left(t \mid \kappa_{i}\right)
\end{aligned}
$$

where $\hat{x}_{j}\left(t \mid \kappa_{i}\right), j \in\{1, \ldots, L\}$ are obtained as follows:

$$
\begin{aligned}
& \hat{x}_{j}\left(t \mid \kappa_{i}\right)=\left[I-K_{\kappa_{i}}^{(j)} C_{\kappa_{i}}^{(j)}\right] \hat{x}_{j}^{-}\left(t \mid \kappa_{i}\right)+K_{\kappa_{i}}^{(j)} h_{i}^{(j)}(\theta(t)) y(t) \\
& \hat{x}_{j}^{-}\left(t+1 \mid \kappa_{i}\right)=A_{\kappa_{i}}^{(j)} \hat{x}_{j}\left(t \mid \kappa_{i}\right)+h_{i}^{(j)}(\theta(t)) B_{\kappa_{i}}^{(j)} u(t)
\end{aligned}
$$

with:

$$
K_{\mathrm{\kappa}_{i}}^{(j)}=P_{\mathrm{\kappa}_{i}}^{(j)}\left(C_{\mathrm{\kappa}_{i}}^{(j)}\right)^{T}\left[C_{\mathrm{\kappa}_{i}}^{(j)} P_{\mathrm{\kappa}_{i}}^{(j)}\left(C_{\mathrm{\kappa}_{i}}^{(j)}\right)^{T}+S_{v}\right]^{-1}
$$

where $P_{\mathrm{K}_{i}}^{(j)}$ is the solution of the discrete Riccati equation:

$$
P_{\mathrm{\kappa}_{i}}^{(j)}=A_{\kappa_{i}}^{(j)}\left[P_{\kappa_{i}}^{(j)}-K_{\kappa_{i}}^{(j)} C_{\kappa_{i}}^{(j)} P_{\kappa_{i}}^{(j)}\right]\left(A_{\kappa_{i}}^{(j)}\right)^{T}+G_{\kappa_{i}}^{(j)} S_{w}\left(G_{\kappa_{i}}^{(j)}\right)^{T}
$$

and the superscript - indicates that the quantity is calculated before the measurement is taken into account.

According to [27], some properties would hold for the LPV Kalman filter (8)-(13) applied to the LPV system (1)(2) when $\kappa=\kappa_{i}$ (i.e. when the real value of the unknown parameter $\kappa$ equals the $i$-th candidate parameter value $\kappa_{i}$ ), among which the fact that $\hat{x}\left(t \mid \kappa_{i}\right)$ would be an unbiased estimation of $x(t)$.

Remark: The choice of the set of candidate parameter values $\left\{\kappa_{1}, \ldots, \kappa_{N}\right\}$ plays an important role in the quality of the estimation $\hat{\kappa}(t)$. In general, it is desirable to consider a dense gridding of the possible values of $\kappa(t)$, i.e. a large number of candidate parameter values, in order to obtain an estimation $\hat{\kappa}(t)$ which is as close as possible to the real value $\kappa(t)$. However, in practice, the benefits of increasing the density of such gridding are limited by the presence of disturbances and noise, which could cause models corresponding to different (but close) values of $\kappa(t)$ to be indistinguishable.

\section{A. Dynamic weighting signal generator (DWSG)}

The dynamic weights $p_{i}(t)$ are generated as follows:

$$
p_{i}(t+1)=\frac{\left.p_{i}(t) \beta_{i}(t)\right) e^{-\omega_{i}(t)}}{\sum_{j=1}^{N} p_{j}(t) \beta_{j}(t) e^{-\omega_{j}(t)}}
$$

where $\beta_{i}(t)$ is a positive weighting matrix function and $\omega_{i}(t)$ is the error measuring function, which maps the measurable signals and the state of the Kalman filter (8)-(13) to a nonnegative real value. The initial conditions $p_{i}(0)$ should be chosen such that $p_{i}(0)>0, i=1, \ldots, N$.

In the following, with the aim of scaling the energy of the estimation error sequences making them comparable, we will use:

$$
\begin{aligned}
& \omega_{i}(t)=\frac{1}{2}\left\|y(t)-\hat{y}\left(t \mid \kappa_{i}\right)\right\|_{S_{\mathrm{K}_{i}}(\theta(t))^{-1}}^{2} \\
& \beta_{i}(t)=\frac{1}{\sqrt{\left|S_{\kappa_{i}(\theta(t))}\right|}}
\end{aligned}
$$

where $S_{i}(\theta(t))$ is a positive definite weighting matrix, and $\|x\|_{S}=\sqrt{x^{T} S x}$.

In [26], it was suggested that a suitable choice for $S_{\kappa_{i}}$ would be the innovation covariance matrix of the Kalman filter. In order to perform a similar choice, $S_{\mathrm{K}_{i}}(\theta(t))$ is selected as:

$$
S_{\kappa_{i}}(\theta(t))=\sum_{j=1}^{L} h_{i}^{(j)}(\theta(t)) S_{\kappa_{i}}^{(j)}
$$

with:

$$
S_{\kappa_{i}}^{(j)}=C_{\kappa_{i}}^{(j)} P_{\kappa_{i}}^{(j)}\left(C_{\kappa_{i}}^{(j)}\right)^{T}+S_{v}
$$




\section{PROPERTIES OF THE LPV MMAE}

This section summarizes the relevant properties of the proposed LPV MMAE. The following proposition shows positiveness and boundedness of the dynamic weights $p_{i}(t)$.

Proposition 1: Assume that $p_{i}(0)>0 \forall i \in\{1, \ldots, N\}$. Then, all the signals $p_{i}(t)$ generated by the DWSG (14) are nonnegative, uniformly bounded and contained in $[0,1]$, with:

$$
\sum_{i=1}^{N} p_{i}(t)=1 \quad \forall t>0
$$

Proof: Taking into account (14), let us compute the evolution of $P(t)=\sum_{i=1}^{N} p_{i}(t)$ :

$$
P(t+1)=\frac{\sum_{i=1}^{N} p_{i}(t) \beta_{i}(t) e^{-\omega_{i}(t)}}{\sum_{j=1}^{N} p_{j}(t) \beta_{j}(t) e^{-\omega_{j}(t)}}
$$

which shows that (19) holds. On the other hand, if $p_{i}(0)>0$, then $p_{i}(t)$ calculated from (14) cannot be negative. Hence, $p_{i}(t) \in[0,1] \forall i \in\{1, \ldots, N\}$ follows necessarily.

On the other hand, the following theorem shows that the dynamic weights $p_{i}(t)$ exhibit convergence if some conditions are fulfilled.

Theorem 1: Let $i^{*} \in\{1, \ldots, N\}$ be an index for the set of candidate parameter values $\left\{\kappa_{1}, \ldots, \kappa_{N}\right\}$, and let $I=$ $\{1, \ldots, N\} \backslash i^{*}$ be an index set. If there exist positive constants $n_{1}, t_{1}, \varepsilon$ and $\varepsilon_{1}$ such that the following conditions hold for all $t \geq t_{1}$ and $n \geq n_{1}$ :

$$
\begin{gathered}
\frac{1}{n} \sum_{\tau=t}^{t+n-1}\left(\omega_{i^{*}}(\tau)+\varepsilon\right)<\frac{1}{n} \sum_{\tau=t}^{t+n-1} \min _{j \in I} \omega_{j}(\tau) \\
\ln \bar{\beta}(t)-\ln \beta_{i^{*}}(t)<\varepsilon_{1}<\varepsilon
\end{gathered}
$$

where:

$$
\bar{\beta}(t)=\max _{j \in I} \beta_{j}(t)
$$

then $p_{i^{*}}(t)$ calculated as in (14) satisfies $p_{i^{*}}(t) \rightarrow 1$ as $t \rightarrow \infty$. Proof: Define:

$$
L_{j}(t)=\frac{p_{j}(t)}{p_{i^{*}}(t)} \quad j \in I
$$

From (14), we have:

$$
p_{i}(t)=p_{i}(0) \prod_{\tau=0}^{t-1} \frac{\beta_{i}(\tau) e^{-\omega_{i}(\tau)}}{\sum_{j=1}^{N} p_{j}(\tau) \beta_{j}(\tau) e^{-\omega_{j}(\tau)}}
$$

from which the following is obtained:

$$
L_{j}(t+n)=\left[\prod_{\tau=t}^{t+n-1} \frac{\beta_{j}(\tau) e^{-\omega_{j}(\tau)}}{\beta_{i^{*}}(\tau) e^{-\omega_{i^{*}}(\tau)}}\right] L_{j}(t)
$$

Then, taking logarithm of both sides leads to:

$$
\begin{aligned}
& \ln \frac{L_{j}(t+n)}{L_{j}(t)}=\sum_{\tau=t}^{t+n-1} \ln \left(\beta_{j}(\tau) e^{-\omega_{j}(\tau)}\right)-\sum_{\tau=t}^{t+n-1} \ln \left(\beta_{i^{*}}(\tau) e^{-\omega_{i^{*}}(\tau)}\right) \\
& \leq \sum_{\tau=t}^{t+n-1} \ln \left(\bar{\beta}(\tau) e^{-\omega_{j}(\tau)}\right)-\sum_{\tau=t}^{t+n-1} \ln \left(\beta_{i^{*}}(\tau) e^{-\omega_{i^{*}}(\tau)}\right) \\
& =\sum_{\tau=t}^{t+n-1}\left(\ln \bar{\beta}(\tau)-\ln \beta_{i^{*}}(\tau)\right)+\sum_{\tau=t}^{t+n-1} \omega_{i^{*}}(\tau)-\sum_{\tau=t}^{t+n-1} \omega_{j}(\tau)
\end{aligned}
$$

If (21)-(22) hold, then from (27) it follows that there exists $\gamma>0$ such that:

$$
\ln \frac{L_{j}(t+n)}{L_{j}(t)} \leq-n \gamma
$$

that means:

$$
L_{j}(t+n) \leq e^{-n \gamma} L_{j}(t)
$$

Since $L_{j}(t) \rightarrow 0$ as $t \rightarrow+\infty \forall j \in I$, then $p_{i^{*}}(t) \rightarrow 1$ as $t \rightarrow+\infty$ due to (19), which completes the proof.

The following corollary provides additional information about the parameter estimate $\hat{\kappa}(t)$ given by (7).

Corollary 1: Assume that the conditions of Theorem 1 hold, and let $\omega_{i}(t)$ be defined as in (15). Then, the parameter estimate $\hat{\kappa}_{i}(t)$ converges to the closest to $\kappa$ as $t \rightarrow \infty$ in the following sense:

$$
\begin{aligned}
& \lim _{t \rightarrow \infty} \hat{\kappa}(t)=\kappa_{i^{*}} \\
& i^{*}=\arg \min _{i \in\{1, \ldots, N\}} \lim _{n \rightarrow \infty} \frac{1}{n} \sum_{\tau=t}^{t+n-1} \omega_{i}(\tau) \quad \forall t \geq 0
\end{aligned}
$$

Proof: Using the fact that $p_{i^{*}}(t) \rightarrow 1$ and that, according to $(21)$ :

$$
\varepsilon<\frac{1}{n} \sum_{\tau=t}^{t+n-1} \min _{j \in I} \omega_{j}(\tau)
$$

for any positive integer $n \geq n_{1}$ and for all $t \geq t_{1}$, we can conclude that (30)-(31) hold.

\section{CONVERGENCE OF THE PARAMETER ESTIMATION}

Corollary 1 has shown that as long as the conditions of Theorem 1 hold, the parameter estimate $\hat{\kappa}$ will converge to $\kappa_{i}^{*}$ (see (30)-(31)). In some way, it can be said that the true value of $\kappa$ is closer to $\kappa_{i}^{*}$ than to any other $\kappa_{i}$, $i \in I$. In the LTI framework considered by [26], this fact has led to the definition of Equivalently Identified Plants (EIPs), as the subsets in the uncertain parameter space which have the property that if the uncertain parameter belongs to that subset, then the parameter estimate $\hat{\kappa}(t)$ will converge to a well defined $\kappa_{i}^{*}$. In particular, [26] has also shown that the EIPs can be determined by comparing the power spectral densities (PSDs) of appropriate signals, which are calculated using the equivalent transfer matrices of the local state observers that provide $\hat{x}\left(t \mid \kappa_{i}\right)$.

However, the extension of this concept to the LPV case considered in this paper is challenging from a mathematical point of view. In fact, in the LPV case, the idea of transfer matrices as equivalent frequency characterizations of the input-output behavior of a given system, does not hold anymore. Also, it can be expected that in many cases the convergence of the parameter estimate $\hat{\kappa}$ to one or another $\kappa_{i}$, thus whether the true value of $\kappa$ belongs to one or another EIP, will depend on the particular trajectory of the varying parameter vector $\theta(t)$.

Nonetheless, it is possible to obtain a necessary condition in terms of PSDs and parametric transfer matrices [28] that will allow determining undecidable sets, i.e. subsets of the parameter space in which we know for sure that the convergence of $\hat{\kappa}$ to a particular $\kappa_{i}$ will depend on the trajectory 
of $\theta(t)$. These necessary conditions will be obtained by analysing the particular case of constant trajectories, i.e. $\theta(t)=\theta \in \Theta$.

Theorem 2: Let the input signal $\eta(t)=[w(t), v(t), v(t+$ 1), $u(t)]^{T}$ be a bounded-spectral sequence with $\operatorname{PSD} \Psi_{\eta}(\omega)$, and let $\Omega$ be the set of the N-tuples $\omega(t)$ of signals $\omega_{i}(t)$, $i=1, \ldots, N$ obtained from (1)-(2) and (5)-(11) with the input signal $\eta$ considering all the admissible trajectories of $\theta(t)$ in $\Theta$. Then, given the index $i^{*} \in\{1, \ldots, N\}$ for the set of candidate parameter values $\left\{\kappa_{1}, \ldots, \kappa_{N}\right\}$, if $\forall \omega(t) \in \Omega$ there exist $n_{1}, t_{1}, \varepsilon$ and $\varepsilon_{1}$ such that (21)-(22) hold, then $\forall \theta \in \Theta$ (compare with [26]):

$$
\Upsilon_{\kappa_{i^{*}, \kappa}}(\theta)<\Upsilon_{\kappa_{i}, \kappa}(\theta) \quad \forall i \in I=\{1, \ldots, N\} \backslash i^{*}
$$

with:

$$
\Upsilon_{\kappa_{i}, \kappa}(\theta)=\operatorname{tr}\left[\frac{1}{2 \pi} \int_{-\pi}^{\pi} \mathbb{H}_{i}\left(e^{j \omega}, \theta\right) \Psi_{\eta}(\omega) \mathbb{H}_{i}\left(e^{j \omega}, \theta\right)^{H} S_{\kappa_{i}}(\theta)^{-1} d \omega\right]
$$

where $\mathbb{H}_{i}(z, \theta)$ is the parametric transfer matrix defined by:

$$
\mathbb{H}_{i}(z, \theta)=\mathbb{C}_{i}(\theta)\left(z I-\mathbb{A}_{i}(\theta)\right)^{-1} \mathbb{B}_{i}(\theta)+\mathbb{D}
$$

with:

$$
\begin{aligned}
& \mathbb{A}_{i}(\theta)=\left(\begin{array}{cc}
A_{\kappa}(\theta) & 0 \\
\tilde{A}_{i}(\theta) & \hat{A}_{i}(\theta)
\end{array}\right) \\
& \mathbb{B}_{i}(\theta)=\left(\begin{array}{cccc}
G_{\kappa}(\theta) & 0 & 0 & B_{\kappa}(\theta) \\
\tilde{G}_{i}(\theta) & 0 & \tilde{K}_{i}(\theta) & \tilde{B}_{i}(\theta)
\end{array}\right) \\
& \mathbb{C}_{i}(\theta)=\left(\begin{array}{ll}
C_{\mathrm{\kappa}}(\theta) & -C_{\kappa_{i}}(\theta)
\end{array}\right) \\
& \mathbb{D}=\left(\begin{array}{ccc}
0 & I & 0
\end{array}\right)
\end{aligned}
$$

where $\tilde{A}_{i}(\theta), \hat{A}_{i}(\theta), \tilde{G}_{i}(\theta), \tilde{K}_{i}(\theta)$ and $\tilde{B}_{i}(\theta)$ are defined as follows:

$$
\begin{aligned}
\tilde{A}_{i}(\theta)= & \sum_{j=1}^{L} h_{i}^{(j)}(\theta) K_{\kappa_{i}}^{(j)} C_{\kappa}(\theta) A_{\kappa}(\theta) \\
\hat{A}_{i}(\theta)= & \sum_{j=1}^{L} h_{i}^{(j)}(\theta)\left[I-K_{\kappa_{i}}^{(j)} C_{\kappa_{i}}^{(j)}\right] A_{\kappa_{i}}^{(j)} \\
\tilde{G}_{i}(\theta)= & \sum_{j=1}^{L} h_{i}^{(j)}(\theta) K_{\kappa_{i}}^{(j)} C_{\kappa}(\theta) G_{\kappa}(\theta) \\
\tilde{K}_{i}(\theta)= & \sum_{j=1}^{L} h_{i}^{(j)}(\theta) K_{\kappa_{i}}^{(j)} \\
\tilde{B}_{i}(\theta)= & \sum_{j=1}^{L} h_{i}^{(j)}(\theta)\left\{K_{\kappa_{i}}^{(j)} C_{\kappa}(\theta) B_{\kappa}(\theta)+\left[I-K_{\kappa_{i}}^{(j)} C_{\kappa_{i}}^{(j)}\right] B_{\kappa_{i}}^{(j)}\right\}
\end{aligned}
$$

Proof: If $\forall \omega(t) \in \Omega$ there exist $n_{1}, t_{1}, \varepsilon$ and $\varepsilon_{1}$ such that (21)-(22) hold, this should hold true also $\forall \omega(t) \in \bar{\Omega} \subseteq \Omega$, where $\bar{\Omega}$ denotes the set of $N$-tuples $\omega(t)$ obtained from (1)(2) and (5)-(11) with the input signal $\eta(t)$ considering all admissible constant trajectories $\theta(t)=\theta \in \Theta$.

Defining $\tilde{y}\left(t \mid \kappa_{i}\right)=y(t)-\hat{y}\left(t \mid \kappa_{i}\right)$, when $\theta(t)=\theta$, we obtain the following from (1)-(2) and (8)-(11):

$$
\left(\begin{array}{c}
x(t+1) \\
\hat{x}\left(t+1 \mid \kappa_{i}\right)
\end{array}\right)=\mathbb{A}_{i}(\theta)\left(\begin{array}{c}
x(t) \\
\hat{x}\left(t \mid \kappa_{i}\right)
\end{array}\right)+\mathbb{B}_{i}(\theta)\left(\begin{array}{c}
w(t) \\
v(t) \\
v(t+1) \\
u(t)
\end{array}\right)
$$

$$
\tilde{y}\left(t \mid \kappa_{i}\right)=\mathbb{C}_{i}(\theta)\left(\begin{array}{c}
x(t) \\
\hat{x}\left(t \mid \kappa_{i}\right)
\end{array}\right)+\mathbb{D}\left(\begin{array}{c}
w(t) \\
v(t) \\
v(t+1) \\
u(t)
\end{array}\right)
$$

where $\mathbb{A}_{i}(\theta), \mathbb{B}_{i}(\theta), \mathbb{C}_{i}(\theta)$ and $\mathbb{D}$ are given by (36)-(39).

The PSD of the signal $\tilde{y}\left(t \mid \kappa_{i}\right)$ is given by:

$$
\Psi_{\tilde{y}\left(t \mid \kappa_{i}\right)}(\omega)=\mathbb{H}_{i}\left(e^{j \omega}, \theta\right) \Psi_{\eta}(\omega) \mathbb{H}_{i}\left(e^{j \omega}, \theta\right)^{H}
$$

Using Parseval's theorem [29] and recalling (15), it can be concluded that (30) with:

$$
i^{*}=\arg \min _{i \in\{1, \ldots, N\}}\left\{\Upsilon_{\kappa_{1}, \kappa}(\theta), \ldots, \Upsilon_{\kappa_{N}, \kappa}(\theta)\right\}
$$

and $\Upsilon_{\kappa_{i}, \kappa}(\theta)$ defined as in (34), is equivalent to (30)-(32), which relate to (21)-(22) through Corollary 1.

Hence, a necessary condition for the existence of $n_{1}, t_{1}, \varepsilon$ and $\varepsilon_{1}$ such that (21)-(22) hold $\forall \omega(t) \in \bar{\Omega} \subseteq \Omega$ is that the index $i^{*}$ in (41) does not depend on the value of $\theta$, which corresponds to condition (33).

Definition 1: Given a bounded-spectral input signal $\eta(t)=[w(t), v(t), v(t+1), u(t)]^{T}$ with $\operatorname{PSD} \Psi_{\eta}(\omega)$ and $\theta \in \Theta$, the set of $\theta$-undecidable values $\mathcal{U}_{\theta}$ is the set of parameter values $\bar{\kappa}$ for which there exist (at least) two indices $i^{*} \neq i^{* *} \in$ $\{1, \ldots, N\}$ such that:

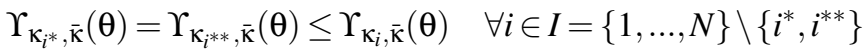

Roughly speaking, if $\bar{\kappa} \in \mathcal{U}_{\theta}$ for a given $\eta$ and $\theta \in \Theta$, then it is not possible to decide whether the closest model to the true one is the model $i^{*}$ or the model $i^{* *}$. Based on this definition, we can state the following corollary of Theorem 3 , which gives a necessary condition for the existence of uniformly decidable sets, i.e. sets of parameter values for which there would be convergence to a single optimal index $i^{*}$, notwithstanding the time-varying parameter $\theta(t)$.

Corollary 2: A necessary condition for a parameter $\bar{\kappa}$ to belong to a uniformly decidable set, i.e. for $\hat{\kappa}(t)$ to converge to a single $\kappa_{i}$ uniformly w.r.t. $\theta(t)$ is that:

$$
\bar{\kappa} \notin \bigcup_{\theta \in \Theta} \mathcal{U}_{\theta}
$$

Proof: The proof is fairly simple. The inclusion $\bar{\kappa} \in$ $\bigcup_{\theta \in \Theta} \mathcal{U}_{\theta}$ implies that $\bar{\kappa} \in \mathcal{U}_{\theta_{o}}$ for some $\theta_{o} \in \Theta$ and hence $\bar{\kappa}$ is $\theta_{o}$-undecidable.

In other words, the fulfillment of (42) is an essential requirement for the existence of an estimate $\kappa_{i *}$ that can be regarded as the closest to the true parameter $\bar{\kappa}$, irrespectively of the trajectory of the time-varying parameter $\theta(t)$.

\section{ILLUSTRATIVE EXAMPLE}

Let us consider a discrete time LPV model as in (1)-(2), with:

$$
\begin{aligned}
A_{\kappa}(\theta(t)) & =\left(\begin{array}{cc}
\kappa & \theta_{1}(t) \\
\kappa \theta_{2}(t) & 0.3
\end{array}\right) & B_{\kappa}(\theta(t)) & =\left(\begin{array}{c}
\kappa \\
5 \theta_{1}(t)
\end{array}\right) \\
C & =\left(\begin{array}{ll}
1 & 0
\end{array}\right) & G_{\kappa}(\theta(t)) & =\left(\begin{array}{c}
\theta_{1}(t) \\
\kappa
\end{array}\right)
\end{aligned}
$$

where $\theta_{1}, \theta_{2} \in[-0.5,0.5]$, and the signals $w(t), v(t)$ have PSD $S_{w}=0.16$ and $S_{v}=0.04$, respectively. In order to 


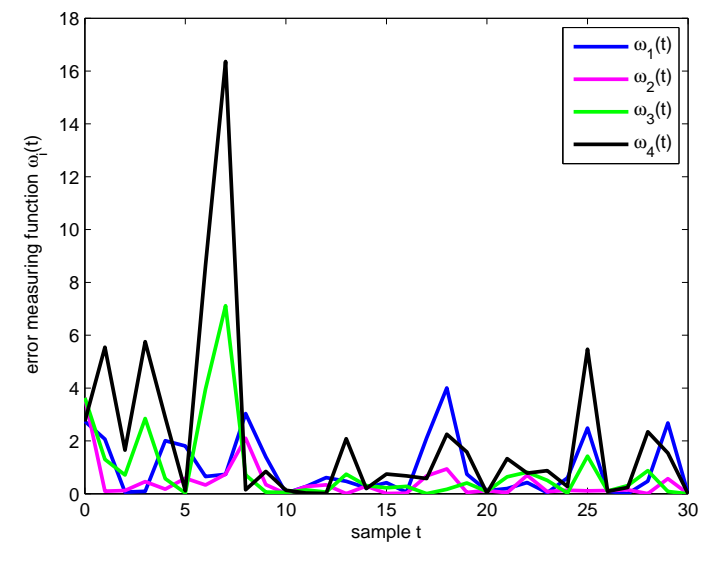

Fig. 1. Error measuring functions in scenario 1.

estimate the state, the set of parameter values $\left\{\kappa_{1}, \kappa_{2}, \kappa_{3}, \kappa_{4}\right\}$ is considered, with $\kappa_{1}=-0.6, \kappa_{2}=-0.2, \kappa_{3}=0.2, \kappa_{4}=0.6$. Then, it can be seen that the representation (3) can be achieved with:

$$
\begin{aligned}
& h_{i}^{(1)}(\theta(t))=\left(0.5-\theta_{1}(t)\right)\left(0.5-\theta_{2}(t)\right) \\
& h_{i}^{(2)}(\theta(t))=\left(0.5-\theta_{1}(t)\right)\left(0.5+\theta_{2}(t)\right) \\
& h_{i}^{(3)}(\theta(t))=\left(0.5+\theta_{1}(t)\right)\left(0.5-\theta_{2}(t)\right) \\
& h_{i}^{(4)}(\theta(t))=\left(0.5+\theta_{1}(t)\right)\left(0.5+\theta_{2}(t)\right)
\end{aligned}
$$

The first simulation (scenario 1) has been performed with $\kappa=-0.2, \theta_{1}(k)=0.5 \sin k$ and $\theta_{2}(k)=0.5 \cos (k / 10)$. The second simulation (scenario 2) has been performed with $\kappa=0.7, \theta_{1}(k)=0.5 \sin (k / 5)$ and $\theta_{2}(k)=0.5 \cos (k / 20)$. The last two simulations have been performed with $\kappa=0$ and the varying parameters chosen as $\theta_{1}(k)=0.5, \theta_{2}(k)=0.5$ (scenario 3 ), and $\theta_{1}(k)=-0.5, \theta_{2}(k)=-0.5$ (scenario 4$)$, respectively. For all simulations, the initial conditions have been chosen as $p_{i}=0.25, i=1,2,3,4$. Also, the input $u(t)$ has been selected as a gaussian white noise with PSD $S_{u}=0.36$.

Fig. 1 shows the response of the error measuring functions in scenario 1 . It can be seen that, on average, $\omega_{2}(t)$ has the smallest energy. This is reasonable, since in scenario 1 , $\kappa=-0.2$, which corresponds exactly to $\kappa_{2}$. As a result, $p_{2}(t)$ converges to 1 , and the remaining dynamic weights converge to 0 , as shown in Fig. 2.

Fig. 3 shows the error measuring functions obtained in scenario 2 . In this case, the function with the smallest energy is $\omega_{4}(t)$. Although $\kappa=0.7 \neq \kappa_{4}=0.6$, the proposed LPV MMAE identifies $\kappa_{4}$ as the unknown parameter value which approximates better the real value of $\kappa$. Hence, the dynamic weight $p_{4}(t)$ is the one that converges to 1, as shown in Fig. 4.

Finally, Figs. 5-6 show the dynamic weights obtained in scenarios 3 and 4, respectively. In this case, the real value of $\kappa$ is between $\kappa_{2}$ and $\kappa_{3}$. In particular, the simulations have shown that $\kappa=0$ belongs to an undecidable set, as defined in Section IV. In fact, different values of the varying parameters

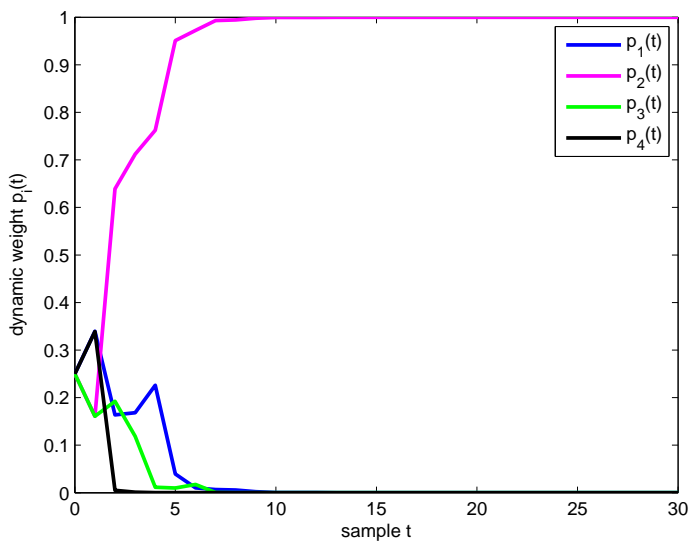

Fig. 2. Dynamic weights in scenario 1.

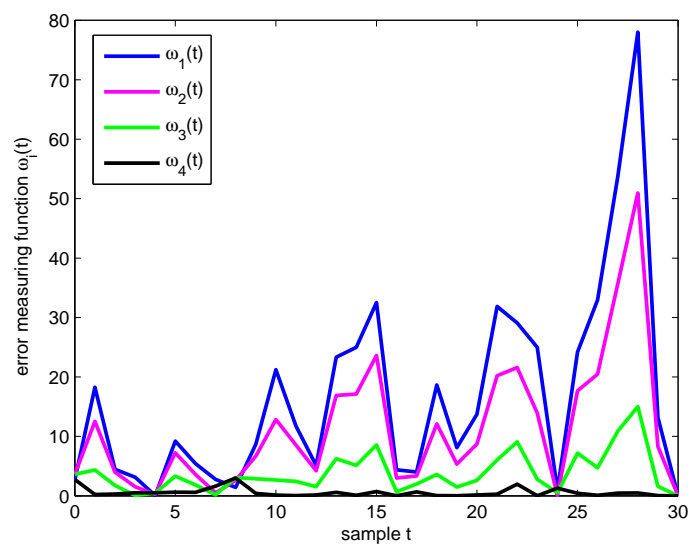

Fig. 3. Error measuring functions in scenario 2 .

$\theta_{1}(t)$ and $\theta_{2}(t)$ correspond to different best models $\left(\kappa_{2}\right.$ in scenario $3, \kappa_{3}$ in scenario 4 ).

\section{CONCLUSIONS}

This paper has presented an MMAE for LPV MIMO systems subject to parametric uncertainty. If some suitable distinguishability conditions hold, then the proposed LPV MMAE will identify the model corresponding to the smallest output prediction error energy. The convergence of the unknown parameter estimation and the existence of undecidable sets have been discussed. Finally, simulation results have been used to illustrate the main properties of the proposed method.

\section{REFERENCES}

[1] Y. Wang, D. M. Bevly, and R. Rajamani, "Interval observer design for LPV systems with parametric uncertainty," Automatica, vol. 60, pp. 79-85, 2015

[2] S. Chebotarev, D. Efimov, T. Raïssi, and A. Zolghadri, "Interval observers for continuous-time LPV systems with $L_{1} / L_{2}$ performance," Automatica, vol. 58, pp. 82-89, 2015.

[3] L. Jaulin, "Nonlinear bounded-error state estimation of continuous time systems," Automatica, vol. 38, no. 2, pp. 1079-1082, 2002.

[4] T. Raïssi, D. Efimov, and A. Zolghadri, "Interval state estimation for a class of nonlinear systems," IEEE Transactions on Automatic Control, vol. 57, no. 1, pp. 260-265, 2012. 


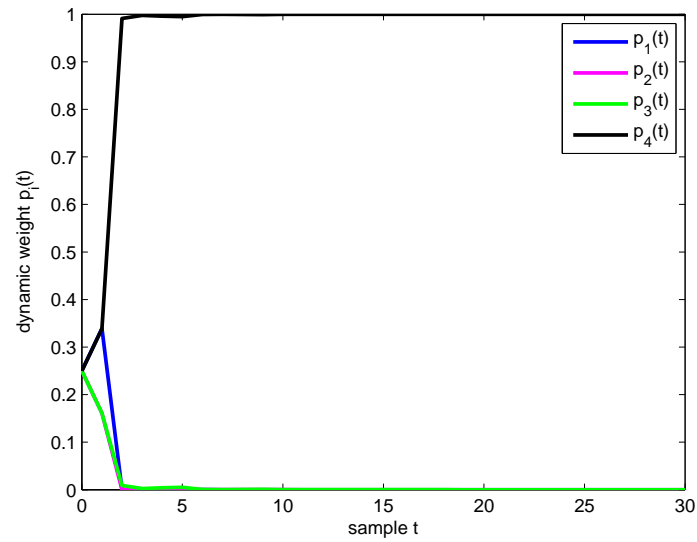

Fig. 4. Dynamic weights in scenario 2.

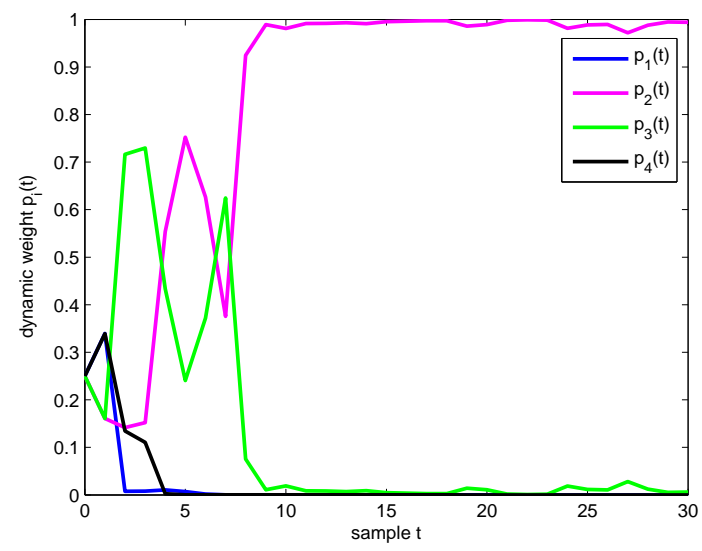

Fig. 5. Dynamic weights in scenario 3.

[5] D. Rotondo, R. Fernandez-Cantí, S. Tornil Sin, J. Blesa, and V. Puig, "Robust fault diagnosis of PEM fuel cells using a Takagi-Sugeno interval observer approach," International Journal of Hydrogen Energy, vol. 41, pp. 2875-2886, 2016.

[6] P. P. Mauridis and G. V. Moustakides, "Simplified Newton-type adaptive estimation algorithms," IEEE Transactions on Signal Processing, vol. 44, no. 8, pp. 1932-1940, 1996.

[7] G. Glentis, K. Berberidis, and S. Theodoridis, "Efficient least squares adaptive algorithms for FIR transversal filtering," IEEE Signal Processing Magazine, vol. 16, no. 4, pp. 13-41, 1999.

[8] D. Rotondo, V. Reppa, V. Puig, and F. Nejjari, "Adaptive observer for switching linear parameter-varying (LPV) systems," in Proc. of the 19th IFAC World Congress, 2014, pp. 1471-1476.

[9] V. Hassani, J. Hespanha, M. Athans, and A. Pascoal, "Stability analysis of robust multiple model adaptive control," in Proc. of the 18th IFAC World Congress, 2011, pp. 350-355.

[10] V. Hassani, A. J. Sørensen, A. M. Pascoal, and A. P. Aguiar, "Multiple model adaptive wave filtering for dynamic positioning of marine vessels," in Proc. of the American Control Conference, 2012, pp. 6222-6228.

[11] L. Giovannini, G. Sanchez, and M. Benosman, "Observer-based adaptive control using multiple-models switching and tuning," IET Control Theory and Applications, vol. 8, no. 4, pp. 235-247, 2014.

[12] A. Cristofaro, T. A. Johansen, and A. P. Aguiar, "Icing detection and identification for unmanned aerial vehicles: multiple model adaptive estimation," in Proc. of the European Control Conference (ECC), 2015, pp. 1651-1656.

[13] M. S. Chong, D. Nešić, R. Postoyan, and L. Kuhlmann, "Parameter and state estimation of nonlinear systems using a multi-observer under the supervisory framework," IEEE Transactions on Automatic Control, vol. 60, no. 9, pp. 2336-2349, 2015.

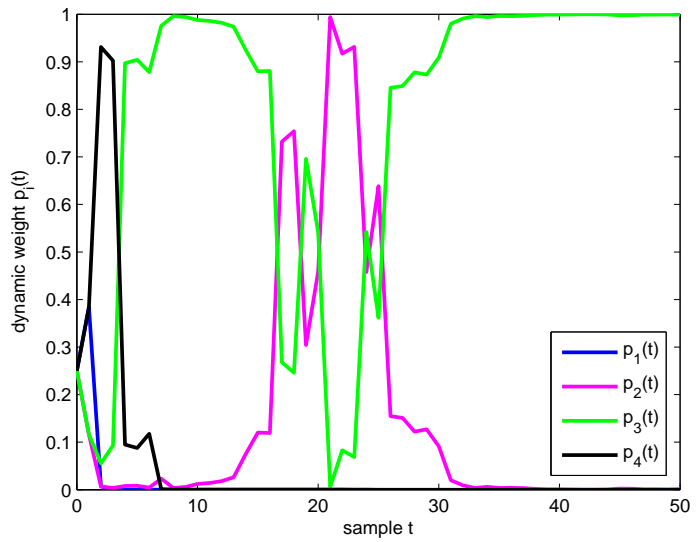

Fig. 6. Dynamic weights in scenario 4.

[14] X.-R. Li and Y. Bar-Shalom, "Multiple-model estimation with variable structure," IEEE Transactions on Automatic Control, vol. 41, no. 4, pp. 478-493, 1996.

[15] C. Hoffmann and H. Werner, "A survey of linear parameter-varying control applications validated by experiments or high-fidelity simulations," IEEE Transactions on Control Systems Technology, vol. 23, no. 2, pp. 416-433, 2015.

[16] J. S. Shamma, "An overview of LPV systems," in Control of Linear Parameter Varying Systems with Applications, J. Mohammadpour and C. Scherer, Eds. Springer, 2012.

[17] D. J. Leith and W. E. Leithead, "On formulating nonlinear dynamics in LPV form," in Proc. of the 39th Conference on Decision and Control, 2000, pp. 3526-3527.

[18] — , "Survey of Gain-Scheduling Analysis Design," International Journal of Control, vol. 73, no. 11, pp. 1001-1025, 2000.

[19] A. Kwiatkowski, M. T. Boll, and H. Werner, "Automated Generation and Assessment of Affine LPV Models," Proceedings of the 45th IEEE Conference on Decision and Control, San Diego, CA, USA, pp. 66906695, 2006.

[20] D. Rotondo, V. Puig, F. Nejjari, and M. Witczak, "Automated generation and comparison of Takagi-Sugeno and polytopic quasi-LPV models," Fuzzy Sets and Systems, vol. 277, pp. 44-64, 2015.

[21] D. Rotondo, A. Cristofaro, T. A. Johansen, F. Nejjari, and V. Puig, "Icing detection in unmanned aerial vehicles with longitudinal motion using an LPV unknown input observer," in Proc. of the IEEE MultiConference on Systems and Control (MSC), 2015, pp. 984-989.

[22] D. Rotondo, V. Puig, F. Nejjari, and J. Romera, "A fault-hiding approach for the switching quasi-LPV fault tolerant control of a four-wheeled omnidirectional mobile robot," IEEE Transactions on Industrial Electronics, vol. 62, no. 6, pp. 3932-3944, 2015.

[23] C. Poussot-Vassal, O. Sename, L. Dugard, P. Gáspár, Z. Szabó, and J. Bokor, "A new semi-active suspension control strategy through LPV technique," Control Engineering Practice, vol. 16, no. 12, pp. 15191534, 2008.

[24] M. Halimi, G. Millérioux, and J. Daafouz, "Model-based modes detection and discernibility for switched affine discrete-time systems," IEEE Transactions on Automatic Control, vol. 50, no. 10, pp. 1567$1580,2005$.

[25] X. D. Sun and I. Postlethwaite, "Affine LPV modelling and its use in gain-scheduled helicopter control," in Proceedings of the UKACC International Conference on Control, 1998, pp. 1504-1509.

[26] V. Hassani, A. P. Aguiar, M. Athans, and A. M. Pascoal, "Multiple model adaptive estimation and model identification using a minimum energy criterion," in Proc. of the 26th American Control Conference (ACC), 2009, pp. 518-523.

[27] D. Simon, "Kalman filtering for fuzzy discrete time dynamic systems,' Applied Soft Computing, vol. 3, pp. 191-207, 2003.

[28] F. Blanchini, D. Casagrande, S. Miani, and U. Viaro, "Stable LPV realization of parametric transfer functions and its application to gainscheduling control design," IEEE Transactions on Automatic Control, vol. 55, no. 10, pp. 2271-2281, 2010.

[29] S. Haykin, Adaptive filter theory. Prentice Hall, 1995. 